\title{
Editorial \\ Study of the Influence of Abiotic and Biotic Stress Factors on Horticultural Plants
}

\author{
Agnieszka Hanaka ${ }^{1, * \mathbb{C}}$, Małgorzata Majewska ${ }^{2}$ and Jolanta Jaroszuk-Ściseł ${ }^{2}$ \\ 1 Department of Plant Physiology and Biophysics, Institute of Biological Sciences, Faculty of Biology and \\ Biotechnology, Maria Curie-Skłodowska University, Akademicka 19, 20-031 Lublin, Poland \\ 2 Department of Industrial and Environmental Microbiology, Institute of Biological Sciences, Faculty of Biology \\ and Biotechnology, Maria Curie-Skłodowska University, Akademicka 19, 20-031 Lublin, Poland; \\ malgorzata.majewska@umcs.pl (M.M.); jolanta.jaroszuk-scisel@umcs.pl (J.J.-Ś.) \\ * Correspondence: agnieszka.hanaka@umcs.pl
}

check for updates

Citation: Hanaka, A.; Majewska, M.; Jaroszuk-Ściseł, J. Study of the Influence of Abiotic and Biotic Stress Factors on Horticultural Plants. Horticulturae 2022, 8, 6. https:// doi.org/10.3390/horticulturae 8010006

Received: 3 November 2021

Accepted: 30 November 2021

Published: 22 December 2021

Publisher's Note: MDPI stays neutral with regard to jurisdictional claims in published maps and institutional affiliations.

Copyright: (C) 2021 by the authors. Licensee MDPI, Basel, Switzerland. This article is an open access article distributed under the terms and conditions of the Creative Commons Attribution (CC BY) license (https:// creativecommons.org/licenses/by/ $4.0 /)$.
In changing environmental conditions, horticulture plants are affected by a vast range of abiotic and biotic stresses which directly and indirectly influence plant condition. Moreover, biomass production or some of the plant metabolites are expected to steadily increase. Such expectations lead to research on the influence of different stressors and their potential modifiers. It is extremely important to have a holistic approach to the processes taking place in a plant that is affected by stress factors. At the same time, the plant can be affected by very diverse factors that mutually affect and shape the plant growth environment, such as the state of water supply-extremely low or, conversely, too high-the impact of low or high temperatures, which in turn cause a state of drought, increased osmotic pressure and salinity.

This Special Issue (SI) was planned with a structure to consider a large range of aspects on stress factors affecting horticultural plants. It is a research summary on the influence of the stress factors on plant growth and the soil parameters. The studies were investigated at the cellular, tissue, organ and whole plant level. Authors described the impact of stress caused by both climate change and human activity resulting in disorder of the optimum temperature (low- and high-temperature stresses), water balance (water and drought stress and irrigation) and the subsequent disturbance of soil parameters. The SI gathers eleven research papers [1-11] and one review [12]. Three papers were dedicated to cold stress, two to salt stress, two to inorganic pollutants such as metals and phosphate (Phi), three to climate change (i.e., high temperature, water and drought stress) and two to irrigation. The subject of the studies were different plant species, i.e., watermelon, lettuce, kale, potato, tomato, grapevine, hops, orchid, strawberry and Buxus megistophylla.

Among the classical parameters used as indicators of plant condition are morphological, anatomical, physiological, biochemical and genetical ones [12]. Physiological, biochemical and anatomical changes occurring in the plant under the influence of stress factors should be especially strongly noticed and analyzed.

In the presented SI, the most frequently applied morphological features were: seed germination, plant growth, leaf and berry area, leaf number, stem diameter and plant dry weight $[1-3,5,7-11]$. Anatomical characteristics were based mainly on the evaluation of epidermis and mesophyll quality and number of cells and chloroplasts [8]. Physiological aspects were focused on water content in leaves, mineral elements (nutritional elements and heavy metals, e.g., $\mathrm{N}, \mathrm{P}, \mathrm{K}, \mathrm{Mg}, \mathrm{Ca}, \mathrm{Mn}, \mathrm{Fe}, \mathrm{Cu}, \mathrm{Na}, \mathrm{Cl}, \mathrm{Co}, \mathrm{Cr}, \mathrm{Ni}, \mathrm{Pb}$ ), photosynthetic pigments content (chlorophylls $a$ and $b$, carotenoids), polyphenolic compounds content (e.g., polyphenols, phenolic acids and flavonoids), soluble sugars, acids and proline contents and plant growth regulators (e.g., abscisic acid, indole-3-acetic acid, gibberellin A3) [1-11]. Biological traits were mostly lipid peroxidation (i.e., content of malondialdehyde; MDA), level of reactive oxygen species (ROS, e.g., $\mathrm{H}_{2} \mathrm{O}_{2}$ and $\mathrm{O}_{2}{ }^{-\bullet}$ ), activity of antioxidative enzymes (e.g., superoxide dismutase, peroxidase and catalase) and content of non-enzymatic 
compounds (e.g., vitamin C and lycopene) [2,4-11]. Additionally, expression of ethyleneresponsive factor genes was analyzed [4]. Some of the papers studied soil properties [1,9]. At the same time, attention was drawn to the close links between hormonal balance and induction of plant immunity, in which regulatory functions are performed by signaling substances (such as salicylic acid, jasmonic acid, ethylene) produced in the pathways of phenolic compounds transformation under the influence of marker enzymes of the phenylpropanoid pathway [12]. Stress factors affect both the uptake of mineral compounds and the process of photosynthesis, which can be monitored based on the biometric parameters and the level of photosynthetic pigments (chlorophyll and carotenoids).

All these elements have been taken into consideration in twelve publications presented in this SI, providing meaningful results revealing a series of events occurring as a consequence of the influence of various environmental factors and explaining the operation of plant defense mechanisms. In the largest number of works, the increase in polyphenols and phenolic acids content and the activity of antioxidant enzymes has been shown because of the action of the abiotic stress factors [1,2,4,9]. Accumulation of proline was observed in salinity stress after chitosan (CTS) application [2], in low temperature stress (kale, Brassica oleracea var. acephala) [3] and in the Phi supply (potato, Solanum tuberosum) [4].

The ideas of the research papers gathered under the titled "Study of the Influence of Abiotic and Biotic Stress Factors on Horticultural Plants" were divided into five sections. They were dedicated to the following fields: (1) achieving better quality of plant material for food production by changes made in the growth conditions, metabolic and genetic modifications; (2) increasing the plant resistance to environmental stresses by application of exogenous compounds of different chemical character; (3) reducing plant stress caused by anthropogenic activity applying non-genetically modified and genetically modified plants (GMP) and (4) mitigating drought stress by irrigation, whereas the main goal of the review paper [12] was to discuss (5) the positive effect of plant growth-promoting microorganisms (PGPM) on horticulture plant performance during drought stress.

\section{(1) Better quality of plant material for food production.}

To achieve better plant material for food production, changes can be made in the plant growth conditions, its metabolism and genetic material [3,5-7].

Manipulations leading to an increase in the stress tolerance of a plant may not always allow us to obtain new varieties with better parameters. Such an example was the research on Vitis vinifera L. varieties [3] in the wine industry, it seems crucial to find the answers to two questions: (1) do the new genotypes of the pathogen-resistant grapevines (new genotypes with low sensitivity to biotic stress) keep good qualities of fruit? and (2) how do the new varieties react to global climate warming? Frioni et al. [3] proved that the production and fruit composition traits during ripening of several new cross-bred pathogen-resistant grapevine varieties (patented and admitted to cultivation) are significantly lower than two $V$. vinifera traditional varieties, Ortrugo and Sauvignon Blanc. In these studies, five white pathogen-resistant varieties (PRV) listed as UD 80-100, Soreli, UD 30-080, Sauvignon Rytos and Sauvignon Kretos were tested. All tested PRV exhibited an earlier onset of veraison and faster sugar accumulation compared to Ortrugo and Sauvignon Blanc. Such effects could suggest an earlier start of the harvest. Therefore, canopy and ripening management strategies must be significantly adjusted compared to the standard practice employed for the parental Sauvignon Blanc. Overall, PRV could perform better in cooler climates, in north-facing hillsides, or at higher altitudes, where their good resistance to mildews could match an adequate grapes' biochemical balance. Moreover, retaining adequate acidity at harvest is crucial to produce high-quality white wines [3].

Not only genetic manipulation leading to obtain the new varieties, using cold-tolerant rootstocks to efficient adaptation plantlets, or regulation of the growth temperature, but also the nearness of other plants can improve growth parameters and fruit quality of the horticultural plants in stress. Such a phenomenon was observed by Karakas et al. [5]. They showed that strawberries, as salt-sensitive plants, reacted strongly to slight or moderate salinity, reducing the crop yield and quality of fruits. Salt stress negatively affected the 
growth, stomatal conductance, electrolyte leakage, contents of chlorophyll, proline, $\mathrm{H}_{2} \mathrm{O}_{2}$, MDA, activity of catalase and peroxidase and content of the health-related compounds such as vitamin $C$ and lycopene. On the other hand, when strawberry seedlings were grown in combination with Portulaca oleracea $\mathrm{L}$. under $\mathrm{NaCl}$ stress condition, not only an increase in weight of the green parts of the plant and the total fruit yield of strawberry plants, but also an improvement in physiological and biochemical parameters were observed. The cultivation of strawberry plants with P. oleracea directly reduced the concentrations of stress metabolites and antioxidant enzyme levels, as well as indirectly contributing to an increase in vitamin $C$ and lycopene contents. Therefore, Karakas et al. [5] suggested the use of $P$. oleracea on the areas with significant salinity as an environmentally friendly method to diminish salt stress.

The plant struggle with stress may be manifested by changes in metabolism and the accumulation of various compounds. For example, kale tolerance to low temperatures is associated with the presence of specialized metabolites such as polyphenols, carotenoids and glucosinolates, which can act not only as protective factors against environmental stress for the plant, but they can also be a source of beneficial compounds for human health [6]. Ljubej et al. [6] observed that a short $(24 \mathrm{~h})$ chilling period $\left(8^{\circ} \mathrm{C}\right)$ was beneficial for the accumulation of phytochemicals in kale. However, freezing temperatures $\left(-8^{\circ} \mathrm{C}\right)$ caused significant stress and decrease in pigments and phytochemical compound levels. The studies suggested that the temperature of kale cultivation should be controlled by producers to achieve production of crops with a high content of health-related compounds [6].

Lu et al. [7] proved that using cold-tolerant rootstocks may be an efficient adaptation strategy for improving stress tolerance in watermelon (Citrullus lanatus (Thunb.), cv. ZaoJia 8424). It was demonstrated that the improved cold tolerance was associated with gourdgrafted watermelons compared to non-grafted (control) plants. Grafted plants accumulated lower levels of ROS, consequently representing enhanced antioxidant activity. Under cold stress, higher chlorophyll and proline contents and lower MDA content were also determined [7].

(2) Exogenous compounds in plant resistance to environmental stresses.

To mitigate stress, growth of horticultural plants can be supported with exogenous substances during agrotechnical treatments such as 24-epi-brassinolide (EBR) on tomato [4], 5-aminolevulinic acids (5-ALA) on Buxus megistophylla [10] and CTS on lettuce (Lactuca sativa L.) [11].

The exogenous EBR used by Heidari et al. [4] as analog of brassinosteroids eliminated the effects of oxidative stress induced by low temperature in cold-sensitive tomato species. 24-epi-brassinolide decreased the ROS content, simultaneously increasing antioxidant enzymes activity, auxin and gibberellin contents, then improved the growth rate of the tomato.

Yang et al. [10] detected that 5-ALA promoted the growth of B. megistophylla, improved plant survival, increased leaf color and enhanced the greening effect. The content of several kinds of mineral nutrient elements, such as nitrogen, phosphate, calcium, magnesium, iron, copper and boron in leaves of B. megistophylla was strongly increased by 5-ALA treatment. Unfortunately, a negative effect was also observed. Under this treatment, accumulation of cadmium, mercury, chromium and lead in roots increased. Luckily, these toxic elements were intercepted in roots without translocation and accumulation in leaves. The activities of antioxidative enzymes and the stress resistance of plants were enhanced. According to the results, 5-ALA, as a specific activator of biochemical pathways, can lead to both favorable and unfavorable alterations in metabolism. Therefore, Yang et al. [10] recommend application of this non-protein amino acid in urban landscapes to improve stress tolerance of ornamental plants.

It has been proven by Zhang et al. [11] that CTS, the classic, widely commercially used elicitor of plant immunity protecting plants against phytopathogens, can be effective in protecting the plant (Lactuca sativa) against the effects of the abiotic factor-excessive salt concentration. Most likely, its direct protective effect under salinity condition was associated 
with the regulation of intracellular ion concentration, controlling osmotic adjustment and increasing antioxidant enzymatic activity (e.g., peroxidase and catalase) in lettuce leaves. Moreover, results of Zhang et al. [11] showed that exogenous CTS could improve plant growth and biomass under salt stress. There is significant evidence that CTS curbed the accumulation of sodium but enhanced the accumulation of potassium in the leaves of NaCl-treated plants. This fact may be important for obtaining better-quality lettuce and supplementing the deficiencies of $\mathrm{K}$ in the human diet [11]. Chitosan, as natural polysaccharide, is a safe and cheap substance promoting plant growth and increasing the biotic and abiotic stress tolerance of plants.

(3) Reduction of plant stress under anthropogenic activity by application of non-genetically modified plants and GMP.

Human beings create habitats unfavorable for the development of plants. This is the result of industrial and agricultural activity. Fortunately, the plants have also learned to deal with this kind of stress. For example, Maleva et al. [8] found the Neottia ovata growing in the young forest community formed during the natural revegetation of the fly ash deposits (fly ash dump of Verkhnetagil'skaya Thermal Power Station). In Russia, this orchid species is included in several regional Red Data Books, and it is especially interesting to gather knowledge of the adaptive characteristics of orchids. The study of orchid adaptive responses to unfavorable factors by Maleva et al. [8] will help to run the process of the introduction of the $N$. ovata into new environments. The adaptive changes in the leaf mesostructure organization, such as an increase in epidermis thickness, the number of chloroplasts in the cell and the internal assimilating surface were found for the first time by Maleva et al. [8]. The orchid population colonizing the fly ash deposits was characterized by a relatively favorable water balance and stable assimilation indexes further contributing to its high viability.

Crop production is expensive in the areas with low phosphorus (P) availability, and for this reason Domatey et al. [2] were looking for other compounds that may serve as a useful source of assimilable P for Solanum tuberosum L. According to this paper, it is possible to apply Phi as fertilizer, only when plants stay resistant to this phosphorus form. Like herbicides, Phi has an inhibitory effect on plant growth. The authors try to combine these phenomena as a hypothetical advantage. Only if plant genotypes are resistant to Phi could it be used both as herbicide to weed control and the source of bioavailable P. Such a solution would be environmentally friendly. Furthermore, Domatey et al. [2] showed significant genotypic variation in tolerance indices among the five tested genotypes (Atlantic, Longshu3, Qingshu9, Longshu6 and Gannong2). Firstly, antioxidant enzyme activities and proline content increased significantly under Phi treatments compared to control without Phi. Secondly, potato genotypes with larger root systems such as Atlantic and Longshu3 were more tolerant to Phi stress than genotypes with smaller root systems (Qingshu9, Longshu6 and Gannong2) [2].

(4) Mitigation of drought stress by irrigation.

Plants require an adequate amount of water during the growing season. Nowadays, this is a rising problem because there is not enough water in many parts of the world, even in areas where such shortages did not occur in the past. Luckily, water limitations can be partially eliminated using various irrigation methods, e.g., by flooding the inter-row [1] or more precisely drip irrigation that supplies water directly to the place where the plant grows out of the soil [9].

Flooding the inter-row is still the most frequently used irrigation method for hop (Humulus lupulus L.) in northern Portugal. Afonso et al. [1] showed that using this type of irrigation to prevent drought stress can worsen the condition of the soil in inter-rows. The irrigation of the hop fields by flooding the inter-row for more than 20 years caused decreased porosity and increased soil bulk density in the $0-10 \mathrm{~cm}$ soil layer in comparison to the 10-20 cm layer. Fortunately, it did not damage the soil structure of ridges, which are the place of nutrient accumulation's gradual uptake by hop plants. Although irrigation and soil 
tillage have damaged the soil structures, they did not create the negative nutrient gradient along the row. Moreover, they refilled water deficiencies during plant development, and the quality of the hop cone yield was sufficient, but on the other hand, the water consumption was too high [1].

Limited water resources force more economical use of water by precise irrigation techniques. It is important to accurately follow the plants' water needs, and correctly predict the moments of deficiency supplementation. New irrigation techniques based on biological parameters and very precise calculations can serve as an adequate solution. It is important to follow exactly the first reactions of the plants to water restrictions to finally be able to use less water [9].

$\mathrm{Ru}$ et al. [9] devoted the article to the above topic. The authors searched a reliable method to easily quantify and monitor the grapevine water status to enable effective manipulation of the water stress of the plants. It was shown that the study on a daily stem diameter variation of grapevine planted in a greenhouse could be helpful to precise irrigation management of plants. The relative daily variation of the grapevine stem diameter from the vegetative stage to the fruit stage was related to different irrigation levels. Both signal intensity calculation of maximum daily shrinkage (SIMDS) and daily increase (SIDI) can be applied as indicators of the moisture status of grapevine and soil. Ru et al. [9] concluded that SIDI was suitable as an indicator of water status of grapevine and soil during the vegetative and flowering stages, whereas SIMDS was suitable as an indicator of the moisture status of plant and soil during the fruit expansion and mature stages. In general, SIMDS and SIDI were very good predictors of the plant water status during the growth stage and their continuous recording can offer the promising possibility of their use in programming automatic drip irrigation of the grapevine [9].

(5) The positive effect of PGPM on plant performance during drought stress.

Trend towards temperature rise contributes to water evaporation and global warming, eventually leading to drought stress in plants. The review paper of Hanaka et al. [12] discussed the positive influence of PGPM on horticulture plant performance during drought stress. Among mechanisms of plant protection by rhizospheric or plant surface-colonizing and endophytic bacteria and fungi are the production of phytohormones, antioxidants and xeroprotectants, and the induction of plant resistance. On one hand, application of various biopreparations containing PGPM seems to be a relatively cheap, easy to apply and efficient method of alleviating drought stress in plants, with implications in productivity and food condition. On the other hand, the vital problems of using biopreparations containing PGPM include limitations in introducing the microbial inoculum to the appropriate conditions and the low repeatability of their activities. Microorganisms that promote plant growth and at the same time induce physical and chemical alterations that result in enhanced tolerance to abiotic stresses, constitute an important separate group called "induced systemic tolerance" (IST). It should be strongly emphasized that a significant enhancement of the protective effect in drought conditions is achieved by using a mixture of bacterial strains (e.g., from genus Bacillus and Serratia), which indicates the synergistic effect of IST strains. It is also very important to emphasize the special role of fungi in protecting plants against drought. Fungi are more tolerant to drought than bacteria and their abundance in soil increases in water-limiting conditions. This is due to the specific fungal growth and traits which allow an intensive soil and plant tissue exploration and colonization and taking water from resources unavailable to other microorganisms.

Studies on the protection of horticultural plants influencing drought stress indicated that the application of well-selected microorganisms can be efficient [12]. Biopreparations should be multicomponent to achieve an appropriate level of microorganism cooperation and the final desired effect. Moreover, the combination of bacterial and fungal strains into one preparation gives even better effectiveness and reliability. Crop specificity should also be taken into consideration. 
Author Contributions: Conceptualization, A.H. and M.M.; writing-original draft preparation, A.H., M.M. and J.J.-Ś.; and writing-review and editing, A.H., M.M. and J.J.-Ś. All authors have read and agreed to the published version of the manuscript.

Funding: This research received no external funding.

Acknowledgments: We gratefully acknowledge all the authors that participated in this Special Issue.

Conflicts of Interest: The authors declare no conflict of interest.

\section{References}

1. Afonso, S.; Arrobas, A.; Rodrgues, M.Â. Twenty-Years of Hop Irrigation by Flooding the Inter-Row Did Not Cause a Gradient along the Row in Soil Properties, Plant Elemental Composition and Dry Matter Yield. Horticulturae 2021, 7, 194. [CrossRef]

2. Dormatey, R.; Sun, C.; Ali, K.; Qin, T.; Xu, D.; Bi, Z.; Bai, J. Influence of Phosphite Supply in the MS Medium on Root Morphological Characteristics, Fresh Biomass and Enzymatic Behavior in Five Genotypes of Potato (Solanum tuberosum L.). Horticulturae 2021, 7, 265. [CrossRef]

3. Frioni, T.; Squeri, C.; Del Zozzo, F.; Guadagna, P.; Gatti, M.; Vercesi, A.; Poni, S. Investigating Evolution and Balance of Grape Sugars and Organic Acids in Some New Pathogen-Resistant White Grapevine Varieties. Horticulturae 2021, 7, 229. [CrossRef]

4. Heidari, P.; Entazari, M.; Ebrahimi, A.; Ahmadizadeh, M.; Vannozzi, A.; Palumbo, F.; Barcaccia, G. Exogenous EBR Ameliorates Endogenous Hormone Contents in Tomato Species under Low-Temperature Stress. Horticulturae 2021, 7, 84. [CrossRef]

5. Karakas, S.; Bolat, I.; Dikilitas, M. The Use of Halophytic Companion Plant (Portulaca oleracea L.) on Some Growth, Fruit, and Biochemical Parameters of Strawberry Plants under Salt Stress. Horticulturae 2021, 7, 63. [CrossRef]

6. Ljubej, V.; Karalija, E.; Salopek-Sondi, B.; Šamec, D. Effects of Short-Term Exposure to Low Temperatures on Proline, Pigments, and Phytochemicals Level in Kale (Brassica oleracea var. acephala). Horticulturae 2021, 7, 341. [CrossRef]

7. Lu, K.; Sun, J.; Li, Q.; Li, X.; Jin, S. Effect of Cold Stress on Growth, Physiological Characteristics, and Calvin-Cycle-Related Gene Expression of Grafted Watermelon Seedlings of Different Gourd Rootstocks. Horticulturae 2021, 7, 391. [CrossRef]

8. Maleva, M.; Borisova, G.; Chikina, N.; Sinenko, O.; Filimonova, E.; Lukina, N.; Glazyrina, M. Adaptive Morphophysiological Features of Neottia ovata (Orchidaceae) Contributing to Its Natural Colonization on Fly Ash Deposits. Horticulturae 2021, 7, 109. [CrossRef]

9. Ru, C.; Hu, X.; Wang, W.; Ran, H.; Song, T.; Guo, Y. Signal Intensity of Stem Diameter Variation for the Diagnosis of Drip Irrigation Water Deficit in Grapevine. Horticulturae 2021, 7, 154. [CrossRef]

10. Yang, H.; Zhang, J.; Zhang, H.; Xu, Y.; An, Y.; Wang, L. Effect of 5-Aminolevulinic Acid (5-ALA) on Leaf Chlorophyll Fast Fluorescence Characteristics and Mineral Element Content of Buxus megistophylla Grown along Urban Roadsides. Horticulturae 2021, 7, 95. [CrossRef]

11. Zhang, G.; Wang, Y.; Wu, K.; Zhang, Q.; Feng, Y.; Miao, Y.; Yan, Z. Exogenous Application of Chitosan Alleviate Salinity Stress in Lettuce (Lactuca sativa L.). Horticulturae 2021, 7, 342. [CrossRef]

12. Hanaka, A.; Ozimek, E.; Reszczyńska, E.; Jaroszuk-Ściseł, J.; Stolarz, M. Plant Tolerance to Drought Stress in the Presence of Supporting Bacteria and Fungi: An Efficient Strategy in Horticulture 2021. Horticulturae 2021, 7, 390. [CrossRef] 